\title{
Dynamical Models of Lead-Acid Batteries: Implementation Issues
}

\author{
Stefano Barsali and Massimo Ceraolo
}

\begin{abstract}
This paper explains how the lead-acid models described in a previous paper can be utilized in practice. Two main issues are opened by that paper: 1) The paper does not supply detailed information on how to identify the several parameters of the proposed models, and 2) it defines a whole family of models, but does not discuss which model of the family is adequate for a given purpose. These two issues are tackled in this paper. For the first issue, the more complex one, two options are proposed and discussed: 1) a complete identification procedure involving extensive lab tests and 2) a simplified one that combines information from lab tests and data supplied by the manufacturer. In addition, further simplifications applicable in cases of batteries belonging to the same family are presented.
\end{abstract}

Index Terms-Batteries, modeling, parameter estimation.

\section{PROPOSED MODEL}

$\mathbf{I}$ $\mathrm{N}$ THIS PAPER, the lead-acid battery model family described in detail in [11] is rapidly reported for reader's convenience.

The model can be represented by an equivalent electric network reported in Fig. 1, along with the dynamic equations representing the charge storage process and the electrolyte heating

$$
\begin{aligned}
& Q_{e}(t)=\int_{0}^{t}-I_{m}(\tau) d \tau \\
& C_{\theta} \frac{d \theta}{d t}=\frac{\theta-\theta_{e}}{R_{\theta}}+P_{s}
\end{aligned}
$$

where

$Q_{e} \quad$ so-called "extracted charge," i.e., the charge that has been actually extracted from the battery starting from a battery completely full (battery full when $t=0$ );

$C_{\theta}$ and $R_{\theta}$ battery thermal capacitance and resistance, respectively;

$P_{s} \quad$ heating power generated inside the battery by conversion from electrical or chemical energy.

It has to be remembered that the resistances $R_{k}$ and capacitances $C_{k}$ shown in Fig. 1 are function of the battery state-of-charge and electrolyte temperature. The equations defining these parameters are reported in the Appendix.

\section{IdentificAtion of Model Parameters by Lab Tests}

In the diagram of Fig. 2 the battery model is globally described as having the environment temperature $\theta_{a}$ and the bat-

Manuscript received September 8, 2000.

The authors are with the Department of Electrical Systems and Automation, University of Pisa, Pisa, Italy.

Publisher Item Identifier S 0885-8969(02)01511-5.

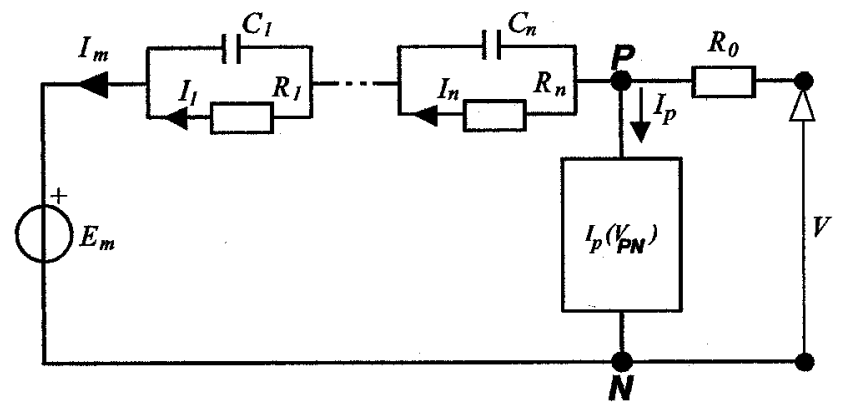

Fig. 1. Lead-acid equivalent network for both discharge and charge.

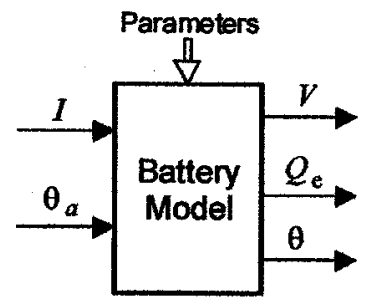

Fig. 2. Pictorial description of model interaction with the external world.

tery current as inputs, and the battery voltage as well as the extracted charge and electrolyte temperature as outputs.

It is in principle possible to determine all the parameters starting from a set of lab tests of a real battery, i.e., discharges and charges at different values of currents and environment temperatures and a digital simulator of the model.

If $V_{m}(t)$ indicates the measured voltage shape of a given test, and $V_{s}(t, \mathbf{p})$ indicates the simulated voltage referring to the same input signals and a given set of parameters $\mathbf{p}$, and err is a measure of the error between the two shapes, the elements of vector $\mathbf{p}$ could in principle be obtained by solving the equation in $\mathbf{p}$

$$
\operatorname{err}\left(V_{m}(t), V(t, \mathbf{p})\right)=\min .
$$

Examples of error functions are

$$
\begin{aligned}
\operatorname{err}(x(t), y(t))= & \sqrt{\frac{1}{T} \int_{t_{1}}^{t_{1}+T}(x(t)-y(t))^{2} d t} \\
& \text { mean quadratic difference. } \\
\operatorname{err}(x(t), y(t))= & \frac{1}{T} \int_{t_{1}}^{t_{1}+T}|x(t)-y(t)| d t \\
& \text { mean absolute difference. }
\end{aligned}
$$

In practice, however, because of the great number of elements of $\mathbf{p}$ and the strong complexity of the relationships between the $p$ elements and the battery behavior, it is very difficult that this 
process converge to reasonable results, if a brute-fore approach is undertaken, i.e., if a general-purpose numerical algorithm is adopted for minimization of (1).

It is therefore advisable to simplify this task, by decomposing the problem into simpler subtasks for which the number of needed parameters is smaller. This is dealt with in the following paragraphs under some assumptions, generally satisfied, on the model structure.

Once the elements of $\mathbf{p}$ are computed, an err function such as those reported in (2) and (3) can be used to check the validity of the results.

\section{A. Parameters Referring to the Battery Capacity}

The battery capacity is defined in the model by the following equation (see also Appendix):

$$
C(I, \theta)_{I, \theta=\mathrm{const}}=\frac{K_{c} C_{0^{*}}\left(1+\frac{\theta}{-\theta_{f}}\right)^{\varepsilon}}{1+\left(K_{c}-1\right)\left(\frac{I}{I^{*}}\right)^{\delta}}
$$

in which $\theta$ is the electrolyte temperature (in ${ }^{\circ} \mathrm{C}$, supposed constant within the battery and during the discharge) and $K_{c}, C_{0^{*}}$, $\theta_{f}, \varepsilon, \delta, I^{*}$ are parameters to be identified.

It is apparent that during real-life lab tests, even if performed in climatic chambers, the electrolyte temperature is different in different electrolyte points and varies along with time as well. For the purpose of interpolation of capacity parameters, however, tests can be made under constant ambient temperature, and the temperature $\theta$ to be considered in (4) is an average of the electrolyte temperature. The average is to be in principle carried out over space and time. In practice the time-average of a single point temperature is sufficient.

This single point temperature, in case of flooded batteries, is the temperature measured in a point chosen so that it reasonably reflects the average electrolyte temperature. For the more complex case of VRLA batteries, experimental tests performed by the authors have shown that for the purpose of the interpolation of parameters of (4) the single-point temperature can be obtained by measuring the temperature of one of the battery pins.

The interpretation of the parameter $I^{*}$ in (4) is quite simple as a consequence: it is a reference current able to make the basis of the power to $\delta$ an adimensional number. It is therefore reasonable to assume $I^{*}=I_{n}$, or, more generally, to equal $I^{*}$ to a value representative of the typical usage of a given battery.

An experimental determination of the remaining five parameters would require, under the hypothesis normally acceptable of assigning to the electrolyte freezing temperature the value $\theta_{f}=-35^{\circ} \mathrm{C}$, at least four tests made using two different (constant) currents $I_{1}$ and $I_{2}$ and two different temperatures $\theta_{1}$ and $\theta_{2}$. If the equation of the capacity is utilized in conjunction with the four couples $\left(I_{1}, \theta_{1}\right),\left(I_{1}, \theta_{2}\right),\left(I_{2}, \theta_{1}\right)$, and $\left(I_{2}, \theta_{2}\right)$ and the related measured capacities, a system of four equations in the four unknowns $C_{0^{*}}, K_{c}, \varepsilon$, and $\delta$ is determined. If more tests are available, the same parameters can be more effectively computed considering all tests and choosing the set of parameters that minimizes the error between measured and computed capacities.

Often the battery manufacturer gives information about the dependency of the capacity on the temperature by means of the

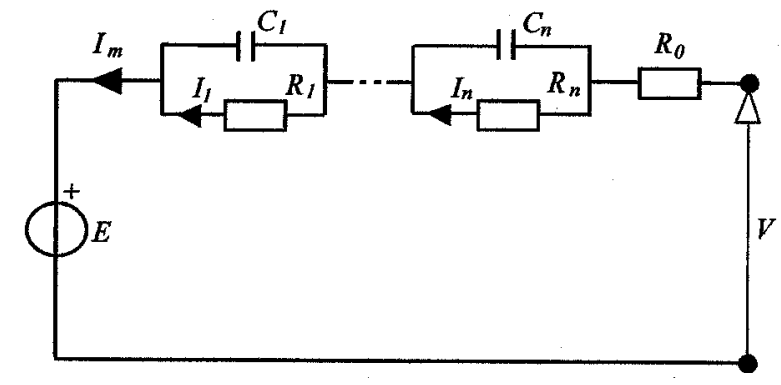

Fig. 3. Lead-acid equivalent network valid during discharge process.

temperature coefficient $\alpha=(1 / C)(\partial C / \partial \theta)$, assumed to be constant.

According to the hypothesis of a constant $\alpha$, to obtain a capacity at a different temperature and equal current of a given value $C\left(I, \theta_{0}\right)$, the simple relation $C(I, \theta)=(1+\alpha \Delta \theta) C\left(I, \theta_{0}\right)$ can be used.

In all cases, the currents and temperatures to be chosen to perform the tests should be representative of the possible operating conditions foreseen for the battery. In fact, even if (4) interpolates quite well the battery behavior under wide ranges of currents and temperatures, it is obvious that for currents and temperatures very far from those used for parameter interpolation nonnegligible errors can occur.

\section{B. Parameters Referring to the Main Branch of the Electric Equivalent}

Consider the generic circuital representation of lead-acid battery reported in Fig. 1.

In [11], that describes the model in detail, it has been stated that the so called Parasitic Reaction Branch, i.e. the branch in the electric circuit reported in Fig. 1 between $\boldsymbol{P}$ and $N$, draws a noticeable current only during the charge process (and at the end of it). Therefore, for the analyzes of tests in which only battery discharge is involved, it can be neglected. This is very useful in that it simplifies a lot the process of parameter identification.

Consider the discharge model represented in Fig. 3, in which, it has to be remembered, the resistances $R_{k}$ and capacitances $C_{k}$ are function of the battery state-of-charge and electrolyte temperature; in addition, each particular battery is characterized by a set of parameters that define the particular shape of these functions

$$
\begin{aligned}
E & =E\left(S O C, \theta, \mathbf{p}_{e}\right) \\
R_{k} & =R_{k}\left(S O C, \theta, \mathbf{p}_{r k}\right) \\
C_{k} & =C_{k}\left(S O C, \theta, \mathbf{p}_{c k}\right)
\end{aligned}
$$

or, if it is assumed $\tau_{k}=R_{k} C_{k}$

$$
\begin{aligned}
E & =E\left(S O C, \theta, \mathbf{p}_{e}\right) \\
R_{k} & =R_{k}\left(S O C, \theta, \mathbf{p}_{r k}\right) \\
T_{k} & =T_{k}\left(S O C, \theta, \mathbf{p}_{t k}\right)
\end{aligned}
$$

where

- $\mathbf{p}_{e}$ is the vector containing the details of the particular battery considered with reference to the electromagnetic force; 


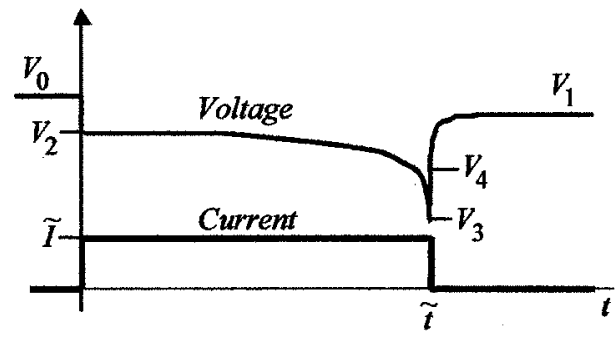

Fig. 4. Typical voltage and current profile for a constant-current discharge.

- $\mathbf{p}_{r k}, \mathbf{p}_{c k}$, and $\mathbf{p}_{t k}$ are vectors containing the details of the particular battery considered with reference to the $k$ th resistance and capacitance, respectively.

In the remainder of this paragraph reference is made to (6) instead of (5).

The identification is particularly simplified if the following conditions can be assumed.

1) Vectors $\mathbf{p}_{e}, \mathbf{p}_{r 0}$ are constituted by two elements each.

2) Vectors $\mathbf{p}_{r k}, \mathbf{p}_{t k}$ with $k>0$ are constituted by a single element.

Under these assumptions, (6) becomes

$$
\begin{aligned}
E & =E\left(S O C, \theta, p_{e 1}, p_{e 2}\right) R_{0}=R_{0}\left(S O C, \theta, p_{r 01}, p_{r 02}\right) \\
R_{k} & =R_{k}\left(S O C, \theta, p_{r k 1}\right) T_{k}=T_{k}\left(S O C, \theta, p_{t k 1}\right) k>0 .(7)
\end{aligned}
$$

in which the numbers at the left hand of the equal signs are to be experimentally evaluated by suitable tests.

A good test is, for instance, is the one pictorially represented in Fig. 4. This test, to be carried out in a thermostatic room, is to be prepared as follows.

1) First, the battery is fully charged.

2) Then, the battery is kept disconnected from any external circuit and the complete stabilization of the voltage is expected (this phase may last several hours).

3) The actual test is composed of measuring of the initial voltage $V_{0}$ (that can be assumed to be equal to the electromotive force corresponding to the condition of battery full and the actual electrolyte temperature).

4) Executing a constant-current discharge for a duration $\tilde{t}$.

5) Registering the subsequent transient up to the complete stabilization of voltage, so that the stabilized voltage $V_{1}$ can be equalled to the battery electromotive force.

The parameters of (7) can be determined as follows.

- $p_{e 1}$ and $p_{e 2}$ can be directly determined by measuring the voltage at the beginning and the end of the test (i.e., $V_{0}$ and $V_{1}$ ) under the assumptions that they are numerically equal to the e.m.f.s at the beginning and the end of the test (the relevant values of $S O C_{\mathrm{beg}}$ and $S O C_{\mathrm{end}}$ can be easily evaluated).

- $p_{r 01}$ and $p_{r 02}$ can be directly determined measuring the "instantaneous" voltage drop during the current fronts, which determine $R_{0 \text {-beg }}=\left(V_{0}-V_{2}\right) / \tilde{I}, R_{0 \text {-end }}=\left(V_{4}-\right.$ $\left.V_{3}\right) / \tilde{I}$ : they are simply obtained by solving the $2 \times 2$ al- gebraic system ${ }^{1}$ :

$$
\left\{\begin{array}{l}
R_{0-\mathrm{beg}}=R_{0}\left(S O C_{\mathrm{beg}}, \theta_{\mathrm{beg}}, p_{r 01}, p_{r 02}\right) \\
R_{0 \text {-beg }}=R_{0}\left(S O C_{\mathrm{beg}}, \theta_{\mathrm{beg}}, p_{r 01}, p_{r 02}\right) .
\end{array}\right.
$$

- $p_{t k 1}, p_{t k 2}$, and $p_{r k 1}$ with $k>0$ can be determined considering the voltage transient after $t=\tilde{t}$ and determining the set of parameters that causes the transient to be best fitted, e.g., minimizing, according to what is stated in Section II, some error measure such as the mean quadratic difference or the mean area difference. The maximum value of $k$ must be chosen according to the considerations reported in Section VI. Therefore, the total drop $V_{1}-V_{4}$ will be shared among the resistances $R_{k}$ with $k>0$. Depending on the order chosen for the model and on the fitting criterion, the value of $V_{4}$, needed to calculate the parameters $p_{r 01}$ and $p_{r 02}$ related to $R_{0}$, will be affected.

It is important to note that in the third order model formulation proposed in [11] the above-reported two conditions that enable the simplified parameter identification are met. In particular, the electrical parameter definition equations are

$$
\begin{aligned}
E_{m} & =E_{m 0}-K_{E}(273+\theta)(1-S O C) \\
R_{0} & =R_{00}\left[1+A_{0}(1-S O C)\right] \\
R_{1} & =-R_{10} \ln (D O C) \quad T_{1}=T_{10}=\mathrm{const}
\end{aligned}
$$

in which, obviously, it can be assumed

$$
\begin{aligned}
& p_{e 1}=E_{m 0} \quad p_{e 2}=K_{E} \\
& p_{r 01}=R_{00} \quad p_{r 02}=A_{0} \\
& p_{r 11}=R_{10} \quad p_{t 11}=T_{10} \text {. }
\end{aligned}
$$

If this formulation is assumed, estimating the electrolyte temperature of (7) is not an issue. In fact, in this case the temperature appears only in the e.m.f., and this equation is utilized with measures taken at the beginning of the discharge (when $V=V_{0}$ ) and at the end of the stabilization period (when $V=V_{1}$ ) so that the electrolyte temperature can be assumed to equal the room temperature.

\section{Parameters Referring to the Parasitic Reaction Branch of the Electric Equivalent}

The parasitic reaction current has been expressed as a function of the parasitic branch voltage $V_{P N}$ and the electrolyte temperature $\theta$

$$
I_{p}=V_{P N} G_{p 0} \exp \left(\frac{V_{P N}}{V_{p 0}+A_{p} \frac{1-\theta}{\theta_{f}}}\right) .
$$

The identification of the constants of this equation, i.e., $G_{p 0}$, $V_{p 0}, A_{p}$ can be obtained by means of tests made when the battery is completely full, and relating the voltage and current at the battery pins and the temperature. In fact, when the battery is completely full it can be assumed that $I_{m} \approx 0$ (see Fig. 1), The electrolyte temperature can be estimated from the ambient temperature.

\footnotetext{
${ }^{1}$ The electrolyte temperature can be measured directly in case of flooded batteries, or (if $\tilde{t}$ is not too large) approximately assumed equal to the temperature of one of the battery pins in case of VRLA.
} 
TABLE I

MANUfACTURER'S DATA FOR THE BATTERIES CONSIDERED IN THIS PAPER

\begin{tabular}{l|c|c|c|c|c|c}
\hline \multicolumn{1}{c|}{ Battery } & $C_{1}[\mathrm{Ah}]$ & $C_{3}[\mathrm{Ah}]$ & $C_{10}=C_{\mathrm{n}}[\mathrm{Ah}]$ & $\theta_{n}\left[{ }^{\circ} \mathrm{C}\right]$ & $V_{\text {eod }}[\mathrm{V}]$ & $\alpha\left[{ }^{\circ} \mathrm{C}^{-1}\right]$ \\
\hline Battery1 & 221 & 366 & 500 & 20 & 1.80 & 0.0085 \\
\hline Battery2 & 227.5 & 357 & 540 & 25 & 1.80 & 0.01 \\
\hline
\end{tabular}

However, several lab tests have shown [6] that it can be assumed, for $V_{m}=2.4 \mathrm{~V} / \mathrm{element}$

$$
I_{p}\left(30^{\circ} \mathrm{C}\right) \approx 10 I_{p}\left(20^{\circ} \mathrm{C}\right) \text {. }
$$

\section{Parameters Referring to the Battery Thermal Model}

The proposed battery thermal model is a simple thermal capacitance-thermal resistance model. These two parameters can be derived experimentally or obtained from the manufacturer.

It must be noted however that, differently from all other parameters, the thermal resistance depends on the installation of the battery in the room. In particular, the relative position of the monoblocks (battery modules) within a battery pack is very important, since it strongly influences the surface in direct contact to the free air surrounding the battery pack.

Approximate estimates of the parameters can be obtained by means of the usual techniques for heat transfer problems, based on the battery mass, shape, case material, etc.

\section{IDENTIFICATION OF MODEl PARAMETERS Using MANUFACTURER'S DATA}

The techniques described in the previous paragraphs for identifying the battery model parameters require several lab tests to be performed.

Obviously, to make such tests some devices able to keep constant the current (e.g., by on-line changing the resistance of a resistor connected to the battery) and the temperature (thermostatic chamber) are needed. Therefore, these tests are costly and complex.

When it is not possible, for technical or economic reasons, to make these tests, an approximate estimate of some of these parameters can be determined with simple computations from the battery data supplied by the manufacturer.

The most readily usable data from the manufacturer are related to the battery capacity.

Normally constant-current discharge capacity at different currents and end-of-discharge voltages are supplied. In addition, the manufacturer often supplies some information on the dependence of the capacity on the temperature, often in terms of a "temperature coefficient" value $\alpha=(1 / C)(\partial C / \partial \theta)$.

If, as often done by manufacturers, $\alpha$ is assumed constant, the following relation can be easily obtained ${ }^{2}$ between $\varepsilon$ and $\alpha$

$$
\varepsilon=\alpha\left(\theta_{n}-\theta_{f}\right) .
$$

The parameter $C_{0^{*}}$ is then obtained by

$$
C\left(I_{n}, \theta_{n}\right)=C_{0^{*}}\left(1+\frac{\theta_{n}}{-\theta_{f}}\right)^{\varepsilon}
$$

2It is sufficient to evaluate $\alpha=(\partial C / \partial \theta) / C$ using the formulation of $C(I, \theta)$ given by (4) and evaluate the resulting formula in the point $\left(I_{n}, \theta_{n}\right)$.
TABLE II

PARAMETERS REFERRING TO CAPACITY FOR THE BATTERIES CONSIDERED IN THIS PAPER

\begin{tabular}{l|c|c|c|c|c}
\hline \multicolumn{1}{c|}{ Battery } & $\theta_{j}\left[{ }^{\circ} \mathrm{C}\right]$ & $\varepsilon$ & $\delta$ & $K_{c}$ & $C_{0 *}[\mathrm{Ah}]$ \\
\hline Battery 1 & -35 & 0.468 & 1.80 & 1.098 & 404.7 \\
\hline Battery 2 & -35 & 0.600 & 1.01 & 1.723 & 390.7 \\
\hline
\end{tabular}

and finally $K_{c}$ and $\delta$ are obtained using values of capacity at currents $I$ different from $I_{n}$

$$
C\left(I, \theta_{n}\right)_{I, \theta=\mathrm{const}}=\frac{K_{c} C_{0^{*}}\left(1+\frac{\theta_{n}}{-\theta_{f}}\right)^{\varepsilon}}{1+\left(K_{c}-1\right)\left(\frac{I}{I^{*}}\right)^{\delta}} .
$$

If a larger number of experimental tests is available it is advisable to exploit all the data available and choose a set of parameters that minimizes the error between measured and computed capacities.

In some cases the manufacturer supplies data about the battery internal resistance. To exploit these data for the parameter identification of the proposed model it is important to know the adopted measuring technique. Two measures are relevant.

- Measure by means of the "instantaneous" voltage drop subsequent to a current step (i.e., something similar to $V_{2}-V_{0}$ or $V_{3}-V_{4}$ in the plot of Fig. 4). In this case, the measure is immediately useful to identify the parameters of $R_{0}$.

- Measure made according to the IEC 896-1 and IEC 896-2 standards. In this case, the result of the measure is not immediately exploitable.

In some cases, however, the IEC resistance can be utilized by means of a trial-and-error simulation cycle:

- use a tentative set of resistance parameters;

- simulate the IEC procedure for measuring the internal resistance, read the IEC resistance on the simulation results;

- correct the tentative parameters;

- repeat the cycle until satisfactorily results are obtained.

Since the proposed thermal model is the simplest dynamic model (linear, first order), when data is available, it is immediately exploitable. If no data is directly available, estimates of thermal capacitance can be drawn considering composition of the battery interior (plates, electrolyte). The internal resistance can be computed starting from information on the physical and geometric characteristics of the battery case.

The thermal time constant (thermal capacitance times thermal resistance) can be experimentally obtained as follows.

- The battery is kept in an oven at a constant temperature (e.g., $10^{\circ} \mathrm{C}$ above the ambient temperature) up to the thermal equilibrium. 
TABLE III

Data of the Discharge Tests for the Assessment of the Parameters of the Electric Components of the two Batteries (SEE Fig. 4)

\begin{tabular}{c|c|c|c|c|c|c|c|c}
\hline Battery & $V_{0}[V]$ & $V_{1}[V]$ & $V_{2}[V]$ & $V_{3}[V]$ & $V_{4}[V]$ & $\theta_{\text {end }}$ & $\widetilde{I}[\mathrm{~A}]$ & $\widetilde{t}[\mathrm{~h}]$ \\
\hline Battery 1 & 2.165 & 1.965 & 2.06 & 1.790 & 1.890 & 26 & 58 & 8.494 \\
\hline Battery 2 & 2.115 & 1.995 & 2.01 & 1.788 & 1.905 & 26 & 63 & 7.197 \\
\hline
\end{tabular}

TABLE IV

PARAMETERS REFERrING TO THE MAIN BRANCH OF THE ElECTRIC EQUIVALENT FOR THE BATTERIES CONSIDERED IN THIS PAPER

\begin{tabular}{c|c|c|c|c|c|c}
\hline Battery & $E_{m 0}[\mathrm{~V}]$ & $K_{E}\left[\mathrm{mV} /{ }^{\circ} \mathrm{C}\right]$ & $R_{00}[\mathrm{~m} \Omega]$ & $A_{0}$ & $R_{10}[\mathrm{~m} \Omega]$ & $T_{10}[\mathrm{~s}]$ \\
\hline Battery 1 & 2.165 & 0.782 & 1.810 & -0.056 & 0.386 & 7200 \\
\hline Battery 2 & 2.115 & 0.832 & 1.667 & 0.236 & 0.647 & 7000 \\
\hline
\end{tabular}

TABLE V

PARAMETERS REFERRING TO THE THERMAL MODEL FOR THE BATTERIES CONSIDERED IN THIS PAPER

\begin{tabular}{l|c|c}
\hline Battery & Thermal capacitance $\left[\mathrm{kJ} /{ }^{\circ} \mathrm{C}\right]$ & Thermal resistance $\left[{ }^{\circ} \mathrm{C} / \mathrm{W}\right]$ \\
\hline Battery 1 & 12 & 1.1 \\
\hline Battery 2 & 20 & 0.6 \\
\hline
\end{tabular}

- Then it is put at the ambient temperature, and the evolution of the electrolyte temperature is registered. ${ }^{3}$

- Based on the registered temperature behavior a best fit time constant can be computed.

\section{EXAMPLES OF PARAMETER IDENTIFICATION}

Some checks of the validity of the proposed battery model have already been presented in [11]. However, in that case, no indication was given on how the numerical parameters used in the model were computed.

Hereafter, on the contrary, the validity of the proposed model is experimentally verified in conjunction with the proposed techniques for identifying the model parameters.

The considered batteries are the same considered in [11], i.e.,

Battery 1: valve-regulated lead-acid (gelled), $C_{10}=500 \mathrm{Ah}$;

Battery 2: flooded lead-acid, $C_{10}=540 \mathrm{Ah}$.

\section{A. Capacity Parameters}

In Section III, a technique to derive some of the model parameters from manufacturer data, thus avoiding specific experimental test is proposed.

The manufacturer's data of interest for the batteries considered in this paper are reported in Table $\mathrm{I}$, in which $C_{1}, C_{3}$, $C_{10}$ are, respectively, the capacity at one, three, ten hour discharge, all measured at the temperature $\theta$ and considering the end-of-discharge voltage $V_{\text {eod }}$ (Volts per element).

Normally the freezing temperature is in the range $(-30,-40)^{\circ} \mathrm{C}$. Considering for both batteries a $\theta_{f}=-35^{\circ} \mathrm{C}$ it is immediately obtained (8): $\varepsilon_{1}=0.468, \varepsilon_{2}=0.600$.

Capacity parameters have been calculated using the three capacity values given by the manufacturer $\left(C_{1}=C\left(I_{1}, \theta_{n}\right), C_{3}=\right.$ $\left.C\left(I_{3}, \theta_{n}\right), C_{10}=C\left(I_{10}, \theta_{n}\right)\right)$ and assessing a fourth value $C\left(I_{10}, \theta_{n}+\Delta \theta\right)$ starting from the knowledge of $\alpha$. The results obtained are reported in Table II.

\footnotetext{
${ }^{3}$ Again, direct measure is possible for flooded batteries, estimates of the electrolyte temperatures are possible using the battery pin temperature.
}

As already noted, these parameters identified from manufacturer's data give only first information on the parameters valid for a given battery. Corrections are expected to be necessary and can be made once the whole set of parameters is identified, and simulations of some experimental test are made, according to what has been said in Section II.

\section{B. Parameters of the Electric Components}

As already noted, it is generally very difficult (or even impossible) to determine the parameters of the electric components starting from manufacturer's data. The process to be followed is therefore necessarily the one described in Section II. This process has been followed on the two batteries considered in this paper, and the results are shown in Tables III and IV.

\section{Parameters Referring to the Battery Thermal Model}

The parameters referring to the battery thermal model have been identified according to the technique suggested in Section II-D: the time constant with the oven test and the capacitance starting from the battery composition and the thermal capacitances of the different battery parts (plates, electrolyte, separator, etc). The results are reported in Table V.

\section{Results}

Using the above parameters some transients have been simulated for both the batteries.

Fig. 5 shows the matching obtained in the test performed for parameter identification according to the rules indicated in Section II-B.

On the other hand, Fig. 6 shows the results obtained by comparing simulation and lab measures using a completely different, highly informative, test case, consisting of a sequence of constant-current $\left(C_{8}\right)$ discharges followed by rest periods in which the current is zero. While the results of Fig. 6 are, obviously, slightly less precise than those of Fig. 5, they are still good.

The interpolation set can be improved by repeated simulations and progressive modifications of some parameters. This however would require, in absence of a specialized, complex computer program, a human operator with knowledge of the mathematical structure of the equation parameters, while in this paper a more automated procedure has been chosen.

Work is in progress to produce a computer program to which sufficient skill is given so that the "repeated simulations and progressive modifications of some parameter" is automated.

\section{SimPLified PARAMETER IDENTIFICATION}

As it has been seen in Section IV, the number of the parameters of the considered battery model, and that in principle should be identified is very large. It has also been seen that only a small 

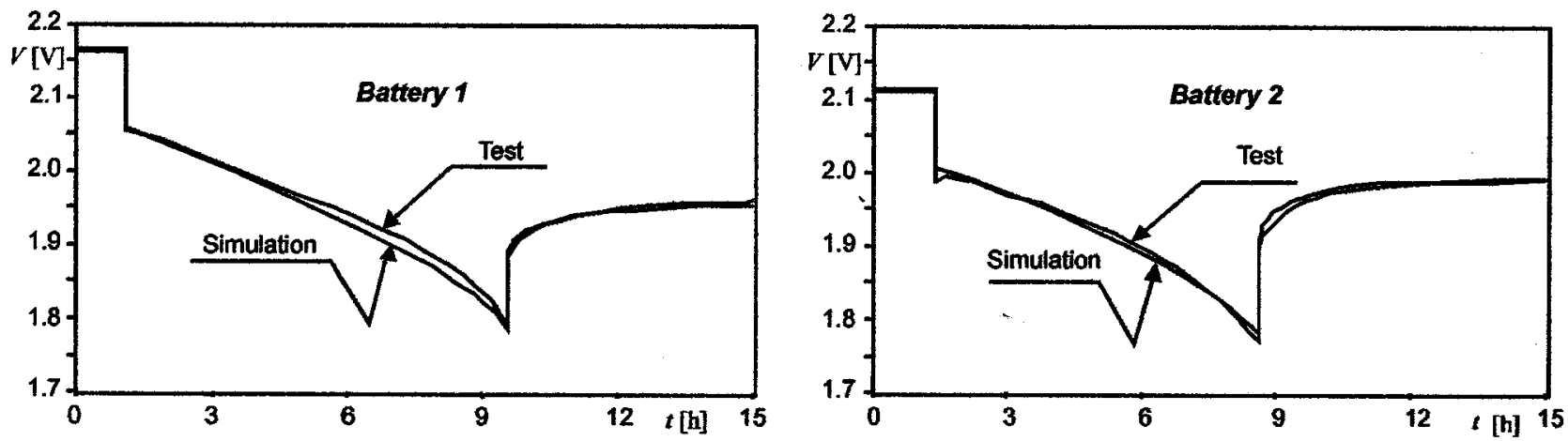

Fig. 5. Comparison between measured and simulated discharge transients for battery 1 and battery 2 (voltages versus time).
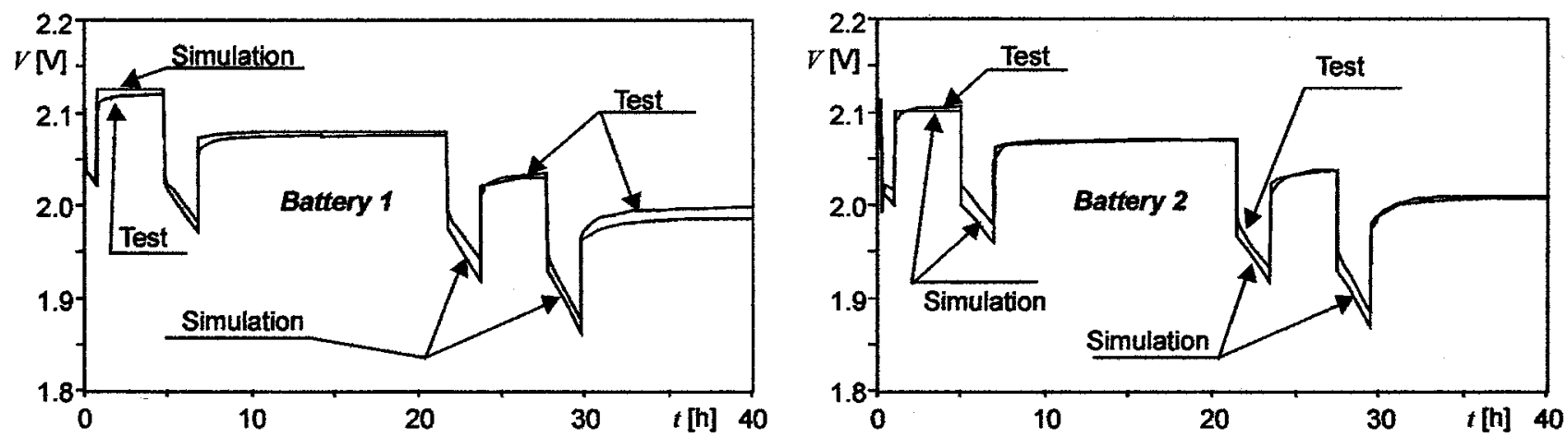

Fig. 6. Comparison between measured and simulated discharge transients for battery 1 and battery 2 (voltages versus time).

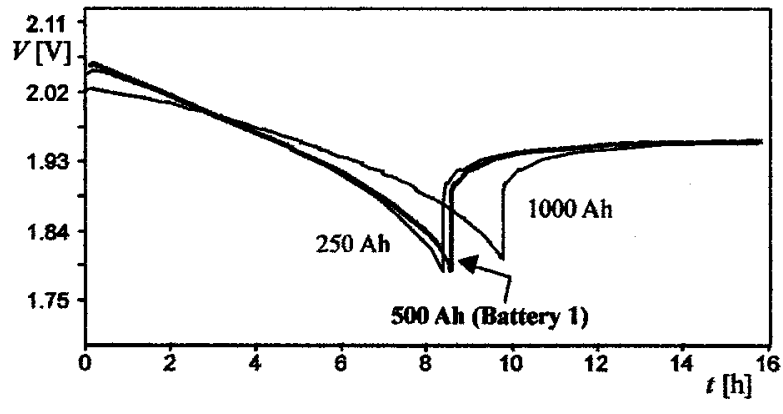

(a)

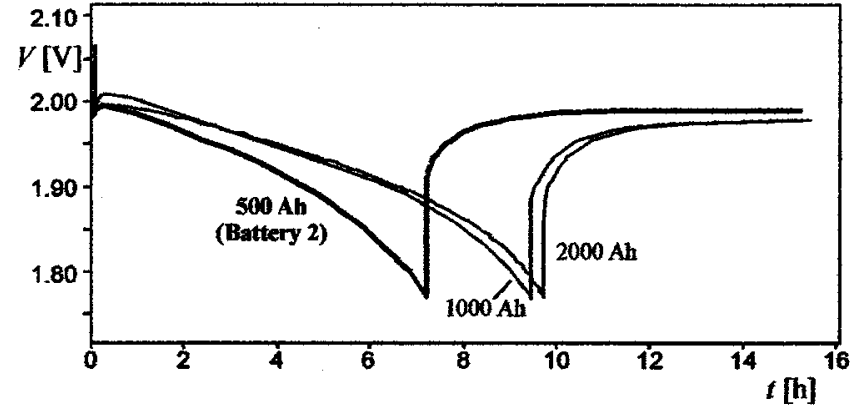

(b)

Fig. 7. Voltage response to a current step (of the type of Fig. 4) of different batteries of the same family. (a) Gelled VRLA of the same manufacturer and model. (b) Flooded of the same manufacturer and model.

part of them can be inferred from the usually published manufacturer's data.

This is an obstacle in the use of the model, although it is an almost automatic consequence of the complexity of the battery behavior.

An idea to reduce the complexity of the parameter identification is to try to exploit similar behavior of batteries having a similar construction.

In Fig. 7, the voltage responses to a current step (of the type of Fig. 4) of different batteries of the same family are shown. The numerical value of the current used is equal to the $C_{8}$ current (current that, according to the manufacturer's documentation, should discharge the batteries in eight hours).

It is easily seen that there exists a nonneglectable dispersion of behavior of different kinds of batteries. However, it looks like all the voltage profiles shown in Fig. 7(a) and (b) can be simulated starting from the parameter interpolations of battery 1 and 2 , and modifying only a very small number of parameters. In fact, making modifications only to parameters $R_{00}, R_{10}, A_{0}$ and $C_{0^{*}}$ and making trivial modifications on the thermal model parameters the behaviors of Fig. 7 can be well matched. Just to give an example, Fig. 8 shows the matching of simulation and experimental data of 1000 Ah batteries using the modified parameters reported in Table VI.

The thermal model parameters have been varied according to these rules.

- The thermal capacitance is taken as proportional to the nominal battery energy $\left(E_{n}=C_{n} \times V_{n}\right)$.

- The thermal resistance is taken as proportional to the battery module external surface. 

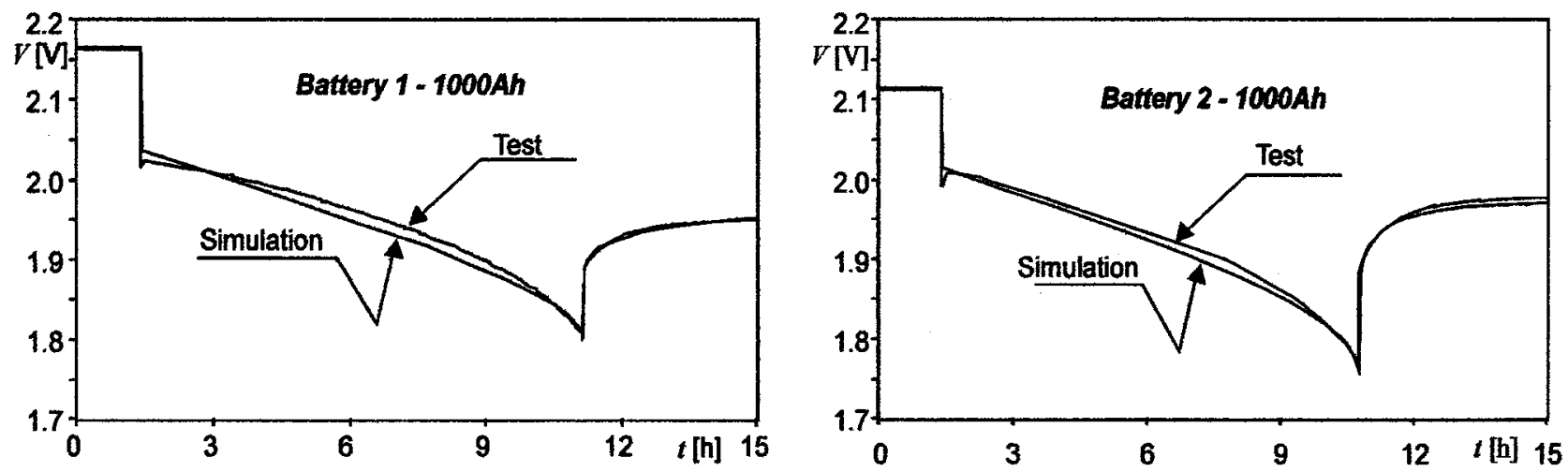

Fig. 8. Comparison of measures and simulations using simplified parameter interpolation (voltages versus time).

TABLE VI

PARAMETERS ModifiEd to MaKe SimUlations Matching EXPERIMENTAL RESULTS WITH MINIMUM IDENTIFICATION EFFORT

\begin{tabular}{l|l|l|l|l}
\hline Battery Type & $R_{00}[\mathrm{~m} \Omega]$ & $R_{10}[\mathrm{~m} \Omega]$ & $A_{0}$ & $C_{0^{*}}[\mathrm{Ah}]$ \\
\hline gelled 500Ah (battery 1) & 1.81 & 0.386 & -0.056 & 404.7 \\
\hline gelled 1000 Ah & 1.10 & 0.150 & -0.40 & 898 \\
\hline flooded 500 Ah (battery 2) & 1.67 & 0.647 & 0.236 & 390.7 \\
\hline flooded 1000 Ah & 0.80 & 0.180 & 0.236 & 868 \\
\hline
\end{tabular}

Therefore, the real number of parameters that has to be identified in practical cases is reduced.

\section{CHOICE OF THE TYPE OF MOdel FOR A PARTICULAR PURPOSE}

The proposed battery models differ to each other by the number of $R-C$ blocks utilized (Fig. 1). The level of accuracy obtainable from different numbers of these blocks can be inferred considering the last part of the voltage transient reported in Fig. 4, i.e., the one after the time $\tilde{t}$.

Fig. 9(a) and (b) shows an example of such a transient as measured in a lab test, compared with the response of models having 1,2 , and $3 R-C$ blocks. The values of $R_{k}-C_{k}$ used for simulations are computed so that the error, measured by a mean quadratic difference (2) is minimized.

The two figures show the same transient, but with different time scales.

It is clear from Fig. 9(a) and (b) that a generic repetition of the experimental behavior in the whole time range, the single exponential interpolation, corresponding to a single $R-C$ block can be acceptable, and the two exponential interpolation appears to be rather good. Also, the details of the voltage response, particularly difficult to follow especially in correspondence of current steps, shows that the three exponential interpolation (three $R-C$ blocks) could be the right choice.

Rather obvious, the more complex the battery model, the more difficult the parameter identification; in addition it must be noted that the repeatability of battery performance is limited; therefore a model that is able to reproduce a set of experimental results, with a high degree of precision, not necessarily will repeat the same performance when other tests are available.

Therefore it is felt that it is useless to consider models having more than three $R-C$ blocks.

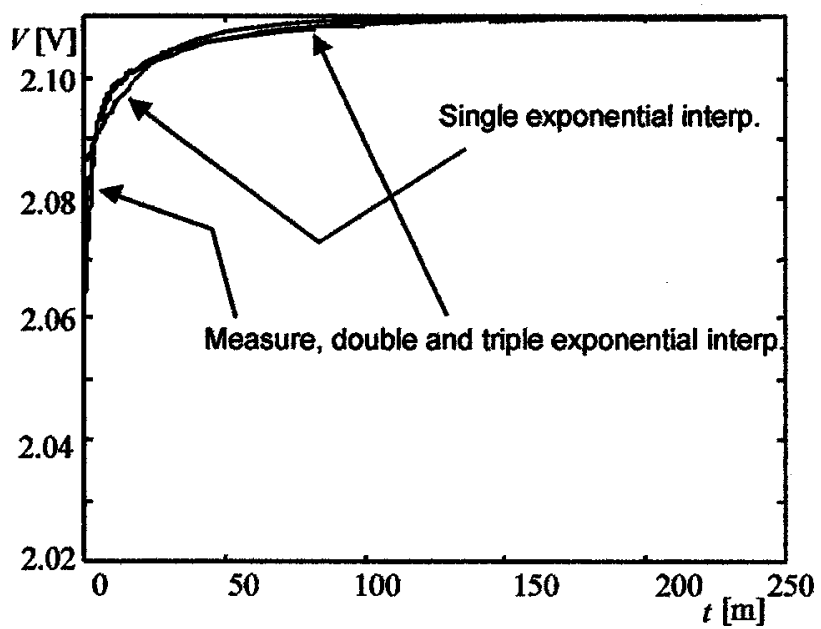

(a)

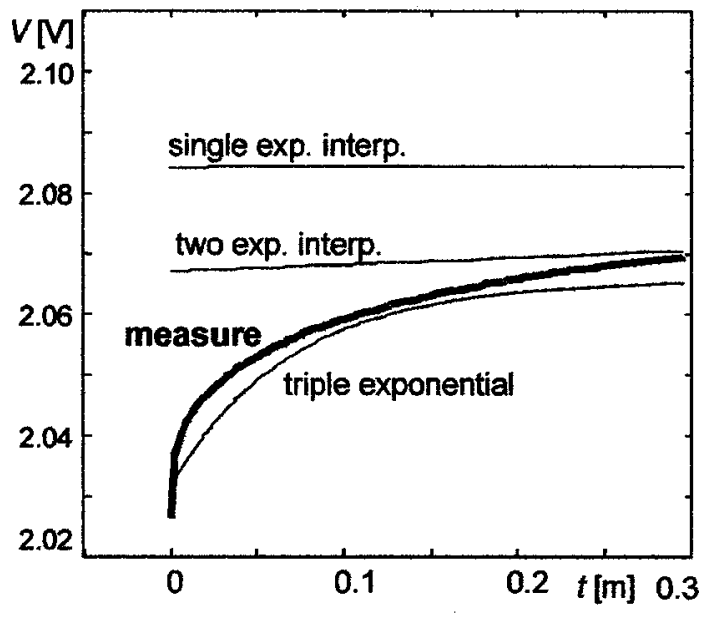

(b)

Fig. 9. Comparison of different degrees of precision of the different considered models (voltages versus time).

\section{CONCLUSION}

- The electrical engineer needs reliable models of the electrochemical batteries, especially lead-acid batteries, to perform the task of system design and simulation. The recent increase in the use of batteries in power systems makes this need stronger.

- In this paper, the practical implications of the utilization of the models presented in [11] are discussed and practical 
information on how to tackle them is supplied. In particular, some simplified ways to identify the model parameters with limited number of lab tests and limited computational effort have been proposed, that still offer a satisfying model accuracy.

- Although an accurate modeling of lead-acid batteries still remains a difficult task, this paper shows that the models proposed in [11], that are a family of models adapted to different levels of precision, give a useful contribution to simplify this task; in particular the third-order formulation, while offering accurate results, is still manageable in terms of computation and parameter identification efforts.

\section{APPENDIX \\ EQuations Defining the Model Parameters}

Battery Capacity as a function of the discharge current $I$ and electrolyte temperature $\theta$

$$
\begin{aligned}
C(I, \theta)= & \frac{K_{c} C_{0^{*}}\left(1+\frac{\theta}{-\theta_{f}}\right)^{\varepsilon}}{1+\left(K_{c}-1\right)\left(\frac{I}{I^{*}}\right)^{\delta}} \\
& \left(K_{c}, C_{0^{*}}, \varepsilon, \delta, I^{*}, \theta_{f} \text { are constant parameters }\right) .
\end{aligned}
$$

State-Of-Charge: $S O C=1-Q_{e} / C(0, \theta)$.

Depth-Of-Charge: $D O C=1-Q_{e} / C\left(I_{\text {avg }}, \theta\right)\left(I_{\text {avg }}\right.$ is the average discharge current).

And, for the third-order formulation

$$
E_{m}=E_{m 0}-K_{E}(273+\theta)(1-S O C) .
$$

Note: Needless to say, $273+\theta$ indicates the electrolyte temperature measured in Kelvin $(\mathrm{K})$.

$$
\begin{aligned}
R_{0} & =R_{00}\left[1+A_{0}(1-S O C)\right] \\
R_{1} & =-R_{10} \ln (D O C) \\
R_{2} & =R_{20} \frac{\exp \left[A_{21}(1-S O C)\right]}{1+\exp \left(\frac{A_{22} I_{m}}{I^{\star}}\right)} \\
C_{1} & =\frac{\tau_{1}}{R_{1}} \\
I_{p} & =V_{P N} G_{p 0} \exp \left(\frac{V_{P N}}{V_{p 0}+A_{p}\left(\frac{1-\theta}{\theta_{f}}\right)}\right)
\end{aligned}
$$

where $E_{m 0}, K_{E}, R_{00}, A_{0}, R_{10}, R_{20}, A_{21}, A_{22}, \tau_{1}, G_{p 0}, V_{p 0}$, $A_{p}, \theta_{f}$ are constant parameters.

\section{ACKNOWLEDGMENT}

The authors wish to thank F. Buonsignori for his continuous effort and his intelligent contribution to all the lab tests on which this paper is based.

\section{REFERENCES}

[1] M. Shepard, "Design of primary and secondary cells, an equation describing battery discharge," J. Electrochem. Soc., July 1965.

[2] K. J. Vetter, Electrochemical Kinetics. New York: Academic, 1967.

[3] H. Bode, Lead-Acid Batteries. New York: Wiley, 1977.

[4] L. Gopikanth and S. Sathyanarana, "Impedance parameters and the state-of-charge," J. Appl. Electrochem., vol. 9, pp. 369-379, 1979.

[5] G. Smith, Storage Batteries. London, U.K.: Pitman, 1980.

[6] R. Giglioli, P. Pelacchi, V. Scarioni, A. Buonarota, and P. Menga, "Battery model of charge and discharge processes for optimum design and management of electrical storage systems," in Proc. 33rd Int. Power Source Symp., Cherry Hill, NJ, June 1988.

[7] H. P. Schoner, "Electrical behavior of lead/acid batteries during charge, overcharge, and open circuit," in Proc. 9th Electric Vehicle Symp. (EVS-9), 1988, Paper N.063.

[8] R. Giglioli, A. Buonarota, P. Menga, and M. Ceraolo, "Charge and discharge fourth order dynamic model of the lead-acid battery," in Proc. 10th Int. Elect. Vehicle Symp., Hong Kong, China, Dec. 1990.

[9] G. Casavola, M. Ceraolo, M. Conte, G. Giglioli, S. Granella, and G. Pede, "State-of-charge estimation for improving management of electric vehicle lead-acid batteries during charge and discharge," in Proc. 13th Int. Elect. Vechicle Symp., Osaka, Japan, Oct. 1996.

[10] M. Ceraolo, D. Prattichizzo, P. Romano, and F. Smargrasse, "Experiences on residual-range estimation of electric vehicles powered by lead-acid batteries," in Proc. 15th Int. Elect. Vehicle Symp., Brussels, Belgium, Sept./Oct. 29-3, 1998.

[11] M. Ceraolo, "Dynamical models of lead-acid batteries," IEEE Trans. Power Syst., vol. 15, pp. 1184-1190, Nov. 2000.

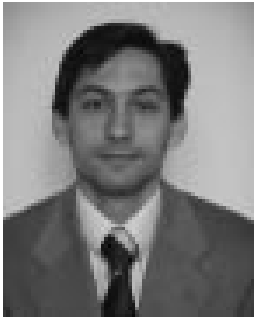

Stefano Barsali was born in Pisa, Italy, in 1969. He received the M.S. and Ph.D. degrees in electrical engineering from the University of Pisa, Pisa, Italy, in 1994 and 1998, respectively.

Currently, he is a Researcher in the field of electric power systems at the University of Pisa. His major fields of interest are electricity market liberalization, dynamic simulation of power systems, distributed generation, and storage batteries.

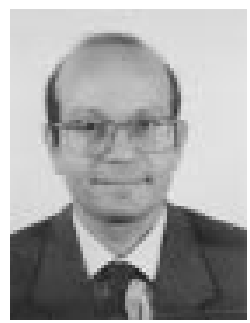

Massimo Ceraolo was born in Castrovillari, Italy, in 1960. He received the M.S. degree in electrotechnical engineering from the University of Pisa, Pisa, Italy, in 1995

Since 1992, he has been working at the University of Pisa as a Researcher and then as an Associate Professor in electric power systems. His major fields of interest include active and reactive compensation of power systems, long-distance transmission systems, computer simulations in power systems, and storage batteries. 